\title{
Classification of Green and Black Teas by PCA and SVM Analysis of Cyclic Voltammetric Signals from Metallic Oxide-Modified Electrode
}

\author{
Nian Liu • Yizeng Liang • Jun Bin • Zhimin Zhang • \\ Jianhua Huang $\cdot$ RuXin Shu $\cdot$ Kai Yang
}

Received: 2 February 2013 / Accepted: 22 May 2013 / Published online: 25 June 2013

(C) The Author(s) 2013. This article is published with open access at Springerlink.com

\begin{abstract}
Forty-three samples of green and black teas were analyzed by an electronic tongue technique. A class of metallic oxide-modified nickel foam electrodes $\left(\mathrm{SnO}_{2}, \mathrm{ZnO}\right.$, $\mathrm{TiO}_{2}, \mathrm{Bi}_{2} \mathrm{O}_{3}$ ) was compared in their sensitivity in this system. The signals obtained by cyclic voltammetry were submitted to multivariate data analysis. In the explorative analysis based on principal component analysis (PCA), the score plots showed that two of these sensors were able to distinguish varieties of teas. The resulting PCA scores were modeled with a support vector machine (SVM) that accomplished final prediction with the qualitative classification of teas. The optimal SVM model was achieved after grid search optimization of some parameters and the conduction of the three commonly used kernel functions. With a comparison of classification accuracies, $\mathrm{Bi}_{2} \mathrm{O}_{3}$-modified nickel foam electrode performed the best among the four electrodes and SVM model using the polynomial kernel attained the highest within the three used kernels. This work demonstrated that cyclic voltammetry combined with the SVM pattern recognition method could be successfully applied in the classification of green and black teas.
\end{abstract}

Keywords Cyclic voltammetry · Chemical sensors . Principal component analysis · Support vector machine . Tea classification

N. Liu $\cdot$ Y. Liang $(\varangle) \cdot J$. Bin $\cdot$ Z. Zhang $\cdot J$. Huang Research Center of Modernization of Chinese Medicines, College of Chemistry and Chemical Engineering, Central South University, Changsha 410083, People's Republic of China

e-mail: yizeng_liang@263.net

R. Shu $\cdot$ K. Yang

Shanghai Tobacco Group Co., Ltd., Shanghai 200082,

People's Republic of China

\section{Introduction}

In food analysis, various analytical methods are used to determine or detect the compound characteristics of food. These methods (Jaimez et al. 2000; Nielsen 2010; Nollet 2000; Simo et al. 2005) show good precision, accuracy, and reliability. However, they demand expensive equipment, complex pretreatment of a sample, and are time-consuming. To overcome these shortcomings, electrochemical methods combined with multivariate data analysis have emerged as rapid and promising tools for evaluation of food quality, especially in the situations where only qualitative or semi-quantitative information is required. This is the so-called electronic tongue, which is mostly for liquid analysis based on the taste sensors, and the first system was presented by Otto and Thomas (1985).

Sensors that have wide selectivity and consequently reveal overlapping signals for different species are used so the data analysis is of great significance in the system. The taste sensors proposed by Toko and his co-workers (Toko 2000) consist of potentiometric electrodes with lipid membranes (PVC membranes with lipid derivatives) and claimed to be capable of discriminating several kinds of foodstuffs such as beer, coffee, and even tomatoes (Kikkawa et al. 1993) with the help of principal component analysis (PCA). Another version of the potentiometric electronic tongue composed of solid-state crystalline ion-selective electrodes based on chalcogenide glass (Vlasov et al. 1994; Vlasov et al. 2000) was applied for the discrimination of beverages (Lvova et al. 2002). The pattern recognition block of that system involved mainly PCA and some kinds of artificial neural networks (ANN) (Vlasov et al. 1997). Winquist's team (Winquist et al. 1997) were pioneers in the development of voltammetric electronic tongues, and the system had also been used to evaluate several food samples (Ivarsson et al. 2001; Winquist et al. 2005) under the combination of PCA. Also, with the 
data analysis methods of PCA and ANN, a hybrid taste sensor based on voltammetric metallic electrodes and potentiometric ion-selective electrodes was employed to classify six types of fermented milk (Winquist et al. 2000). In general, the electronic tongue has been widely used year by year, and it has played a significant role in many aspects (del Valle 2012; Escuder-Gilabert and Peris 2010; Laura EscuderGilabert $^{\mathrm{a}}$ 2010; Riul et al. 2010).

In the classification of tea by electronic tongues, different devices have been constructed for this purpose. A comparison of different applied waveforms on discriminating green and black teas by the pulse voltammetric taste sensor was also studied (Ivarsson et al. 2001). A disposable all-solid-state potentiometric taste sensor coupled with PCA and Soft Independent Modeling of Class Analogy (SIMCA) was investigated in the identification of Korean green tea (Lvova et al. 2003). A combination of the amperometric electronic tongue and linear discriminant analysis (LDA) was utilized to evaluate five classes of tea (Scampicchio et al. 2006). In recent years, several varieties of the Longjing tea were discriminated by the electronic tongue based on multifrequency large amplitude pulse voltammetry (Tian et al. 2007), and a single batch production of Kangra orthodox black tea was classified successfully by a novel impedance tongue (Bhondekar et al. 2011). These studies mentioned above reveal that the taste sensor technique was able to differentiate tea samples, but few studies have focused on the cyclic voltammetric (CV) signals of tea infusion, in spite of cyclic voltammetry's significant place in electrochemical analysis.

Moreover, considering that data analysis is a fundamental part of the electronic tongue system, as we have mentioned above, several pattern recognition techniques such as PCA, LDA, SIMCA, ANN (Lvova et al. 2003; Scampicchio et al. 2006; Vlasov et al. 1997; Winquist et al. 2000) have been explored for the data analysis of the sensors. In the last two decades, a classification algorithm called support vector machine (SVM) (Burges 1998; Shawe-Taylor and Cristianini 2000), which is on the basis of statistical learning theory (Smola and Schölkopf 1998; Vapnik 1998), has been proposed in the broad learning field (Brereton and Lloyd 2010; Li et al. 2009). Despite this success story, few literature dealing with the applications on SVM in the electronic tongue area have been reported (Bhondekar et al. 2011; Ciosek et al. 2006; Kumar et al. 2012; Wei and Wang 2011), furthermore, few of them have combined the cyclic voltammetric method with the utilization of SVM.

In this work, we report an electronic tongue for the classification of green and black teas with multivariate data analysis of cyclic voltammogram of a homemade metallic oxide $\left(\mathrm{SnO}_{2}, \mathrm{ZnO}, \mathrm{TiO}_{2}, \mathrm{Bi}_{2} \mathrm{O}_{3}\right)$ modified nickel foam electrode. Several different combinations of sensors were compared in their classification ability in this system. Using the electrochemical responses obtained from cyclic voltammetry as input information, supervised and unsupervised chemometric analysis methods, principal component analysis (PCA) was performed as the exploratory method and SVM as the classification method.

\section{Materials and Methods}

\section{Materials}

A total of 43 tea samples investigated in this work, from ten provinces in China, were all purchased in the local markets in the city of Changsha, Hunan province. These teas can be divided into two groups, 16 black teas and 27 green teas.

\section{Sample Preparation}

In the experiment, $5.00 \mathrm{~g}$ of tea leaves was prepared as a sample. Before data acquisition of the taste sensors, a sequence of sample preprocessing (He et al. 2009; Ivarsson et al. 2001) was carried out as follows: first, each sample was infused with $100 \mathrm{~mL}$ distilled boiling water for $20 \mathrm{~min}$; second, the tea leaves were removed by a sieve; third, the sample liquid was cooled in a $25^{\circ} \mathrm{C}$ water bath until the temperature reached $25^{\circ} \mathrm{C}$ (approximately $15 \mathrm{~min}$ ). Voltammetric measurements of the tea samples were implemented after an addition of an adequate amount of $2 \mathrm{~mol} \mathrm{~L}^{-1} \mathrm{NaOH}$ solution to yield a final concentration of $1 \mathrm{~mol} \mathrm{~L}^{-1}$ (Novakowski et al. 2011).

\section{Electrodes and Measurements}

The four electronic electrodes were based on nickel foam (from Liyuan New Material Co., Ltd., Changsha, China), which was used as substrate (Hu Hai et al. 2007). The SEM images (obtained from JSM-6360LV scanning electron microscope) of the nickel foam is shown in Fig. 1; it is obvious that the foam has an open cell structure, in which reactants are able to diffuse inside the bulk and the sensitivity of the electrode would be improved to a large extent. The substrate was modified by adding components as nanoparticles of $\mathrm{SnO}_{2}, \mathrm{ZnO}, \mathrm{TiO}_{2}, \mathrm{Bi}_{2} \mathrm{O}_{3}$ (provided by the Sinopharm Chemical Reagent Co., Ltd., Shanghai, China)—one component per sensor. More specifically, every electrode was made by mixing the metal oxide with acetylene black (from Alfa Aesar) and polytetrafluoroethylene (from Dupont de Nemours) liquid (5 wt.\%) in the following weight ratios, $80: 15: 5$, and then rolling the mix into a homogenous paste. The paste was spread onto the nickel foam collectors and finally pressed under $10 \mathrm{MPa}$.

Electrochemical experiments were performed in a CHI600A electrochemical workstation (form ChenHua Instrument Co., Ltd., Shanghai, China) using a conventional three-electrode cell, which was formed by the modified 


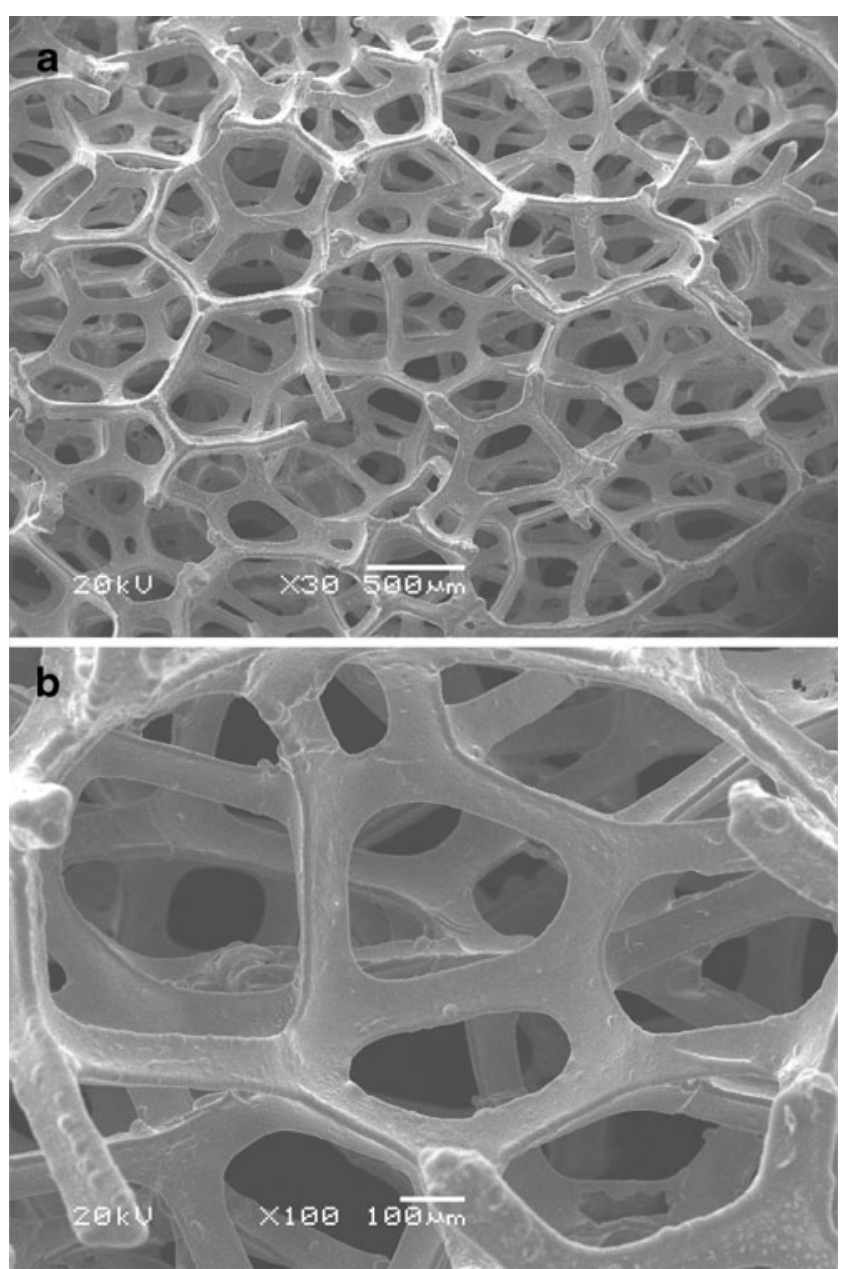

Fig. 1 SEM images of nickel foam

nickel foam working electrode plus the saturated calomel electrode (SCE) reference electrode and a homemade platinum counter electrode. Cyclic voltammograms were registered from -0.7 up to $0.6 \mathrm{~V}$ at a scan rate of $0.01 \mathrm{~V} / \mathrm{s}$. The electrochemical experiments were carried out at a constant temperature $\left(25^{\circ} \mathrm{C}\right)$.

Electrodes were conditioned by running cyclic voltammograms in $1 \mathrm{~mol} \mathrm{~L}^{-1} \mathrm{NaOH}$ solution to attain a stable voltammetric response before performing the measurements with tea samples. In random order, each sample was measured three times and then averaged into one plot.

\section{Statistic Analysis}

A non-supervised multivariate method, principal component analysis (PCA) (Wold et al. 1987) was used to analyze signals obtained from the electronic tongue. The normalized anodic waves constituted the input data matrix for the multivariate analysis. A PCA model was constructed using all samples. The score plot of PC1 versus PC2 was examined for clustering results relating to the two groups, green tea and black tea.
As PCA can be used to reduce the dimension of cyclic voltammogram signals of different kinds of teas, PCs which occupied $98 \%$ of the total information were analyzed by SVM to establish the classification model for green and black tea. Support vector machine algorithm, a powerful multivariate technique based on a machine learning approach, was first introduced by Vapnik and Burges (Burges 1998; Vapnik 1998). In very simple terms, the basic concept of SVM is mapping input vectors to a higher dimensional feature space with kernel tricks, then a maximal margin separating hyperplane is constructed for classification. More details about the theory of SVM can be found in the literature (Webb 2003). Basically, for a given data set $X_{i} \in R^{N}(I=1, \ldots, N)$ with corresponding labels $y_{i}\left(y_{i}=+1\right.$ or -1 , representing the two classes to be classified), SVM gives a decision function:

$f(x)=\sum_{i=1}^{N} y_{i} a_{i} K\left(x_{i}, x_{j}\right)+b$,

where $K\left(x_{i}, x_{j}\right)$ is the kernel function, $x_{i}$ is the input vector, parameter $a_{i}$ is the Lagrange multiplier called support value, $b$ is the bias term. There are three most commonly used kernel functions, which are the linear kernel of $K\left(x_{i}, x_{j}\right)=x_{i}{ }^{T} x_{j}$, the polynomial kernel of $K\left(x_{i}, x_{j}\right)=\left(\gamma x_{i}{ }^{T} x_{j}+r\right)^{d} \gamma>0(d=3$ in this work), and the Gaussian radial basis function (RBF) kernel of $K\left(x_{i}, x_{j}\right)=\exp \left(-\gamma\left\|x_{i}-x_{j}\right\|^{2}\right), \gamma>0$. All of the three kernels were conducted and compared in the present investigation. In addition, when considering the trade-off between the empirical error and the regularized term, penalty parameter $c$ should be taken into consideration. Increasing $c$ means giving more significance to the errors on the training set in determining the optimal hyperplane and leads to the more complex network structures with a smaller separation margin. Therefore, optimization of the modeling parameters is of considerable importance for the development of SVM models. For actual implementation, we used the LIBSVM (version 2.81) package (Chang and Lin 2011), and the penalty parameter $c$ and kernel parameter were tuned based on the training set using the grid search strategy in LIBSVM.

All computations and chemometric analysis were performed in MATLAB version 7.5.0 (MathWorks, Natick, USA).

\section{Result and Discussion}

\section{Electrochemical Characterization of Tea Samples}

Tea has many different varieties because of different processing steps. Green tea, which is brewed from unfermented leaves, contains a rich source of monomer flavanols, while full fermentation to black tea produces a series of theaflavins and thearubigins (Harbowy et al. 1997). These phenolic 
compounds have shown antioxidant abilities in quenching reactive oxygen species and chelating metal ions in diverse tests (Dufresne and Farnworth 2001; McKay and Blumberg 2002). On the other hand, electrochemical analysis can provide valuable information on the physicochemical characteristics of compounds and has been utilized to estimate polyphenols (Ferreira et al. 2006; Freire et al. 2003; Gay Martín et al. 2012; Ghasemi-Varnamkhasti et al. 2012; Gutés et al. 2005; Gutes et al. 2005; Gutiérrez et al. 2008; Novak et al. 2009; Roginsky et al. 2003; Wang et al. 2012). Thus, the first step in this work was the analysis of electrochemical behavior of the teas.

Several cyclic voltammograms obtained by four metal oxide-modified electrodes on two tea samples, as an example of the total 43 samples examined, are shown in Fig. 2. To a certain degree, the four electrodes have similar responses towards these two teas. For instance, most of the $\mathrm{CV}$ diagrams exhibit two redox processes at ca. 0.05 and $0.52 \mathrm{~V}$ (versus $\mathrm{SCE}$ ) in their anodic part, and an evident evolution of the
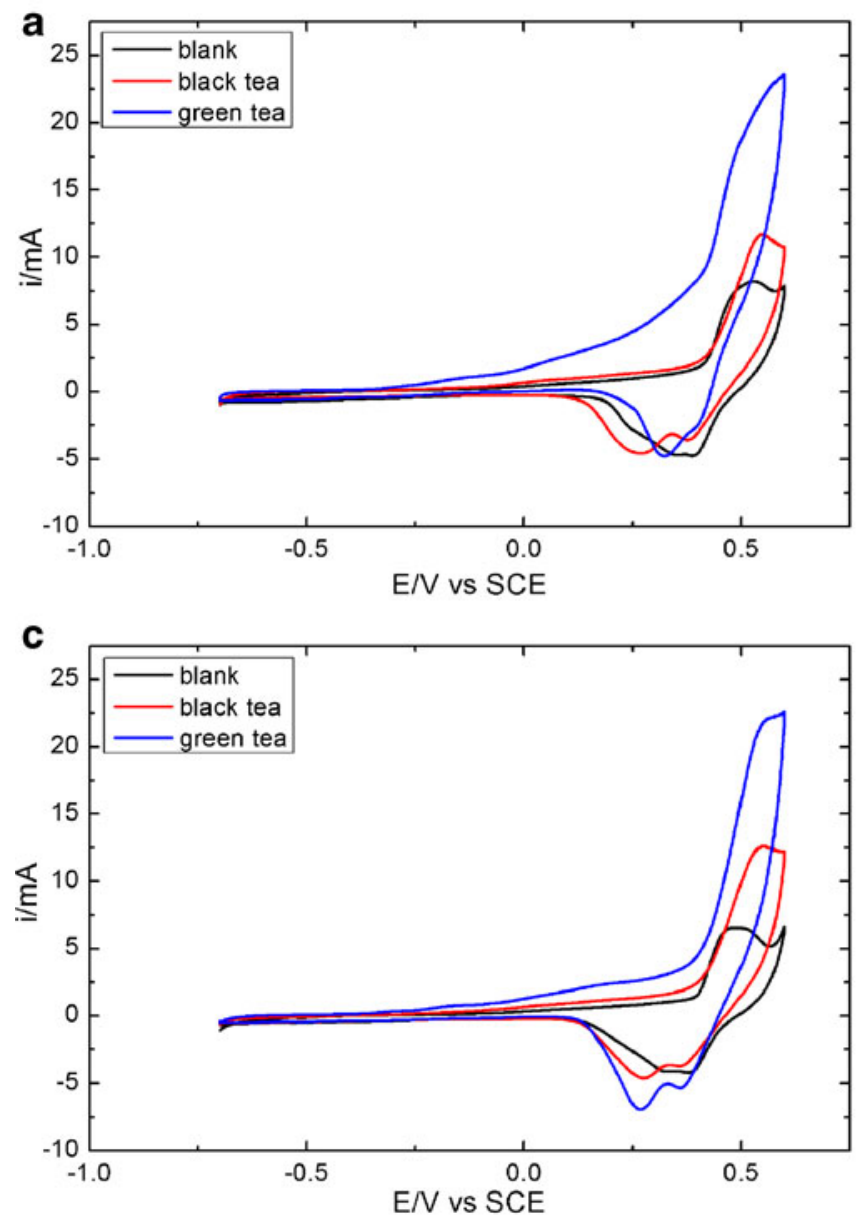

Fig. $2 \mathrm{CV}$ scans recorded on different kinds of teas. a $\mathrm{SnO}_{2}$-modified nickel foam electrode performed as the working electrode. $\mathbf{b} \mathrm{TiO}_{2}-$ modified nickel foam electrode performed as the working electrode. $\mathbf{c}$ signal, increasing in peak current, is detectable over the different teas at $0.52 \mathrm{~V}$. More specifically, a blank $\mathrm{NaOH}$ solution $\left(1 \mathrm{~mol} \mathrm{~L}^{-1}\right)$ gets the lowest, while green tea's peak current is higher than that of black tea. Nevertheless, according to previously published studies (Roginsky et al. 2003), these peaks are in relation with the polyphenolic content of the samples. To be specific, different teas contain different phenolics in different contents. The response of green tea is dominated by epigallocatechin gallate (EGCG), while theaflavins make up the bulk of the phenolics in black tea; previous studies (Kilmartin and Hsu 2003; Roginsky et al. 2003) have shown that EGCG has higher intensities than theaflavins in cyclic voltammetry. Besides that, the overall response would be the sum of the various substances, and the total flavonoids' content of green tea is higher than that of black tea. In the corresponding reverse scan, the cathodic wave of redox processes appears at 0.32 and $0.40 \mathrm{~V}$ (versus SCE). Though different electrodes under study show similar redox processes, their responses differ in the shapes of the diagrams and the

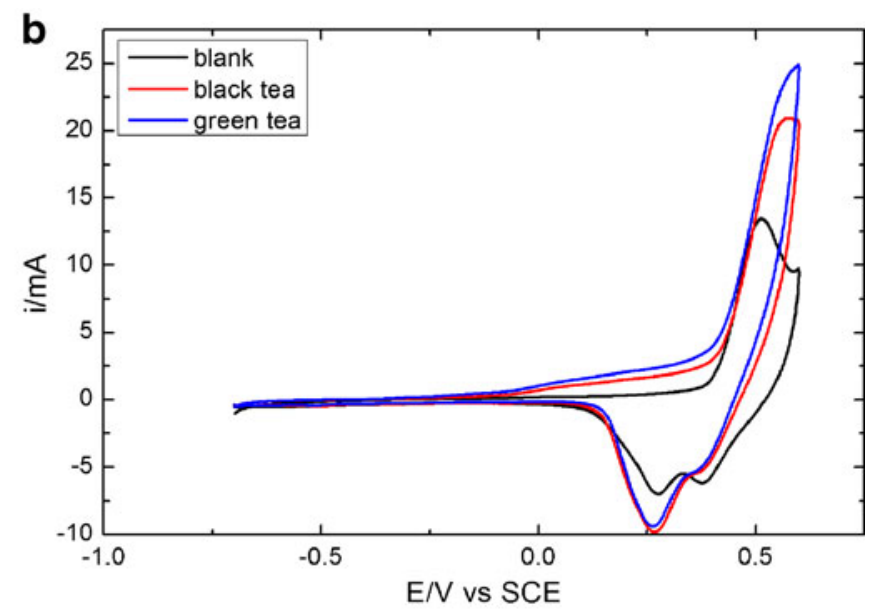

d

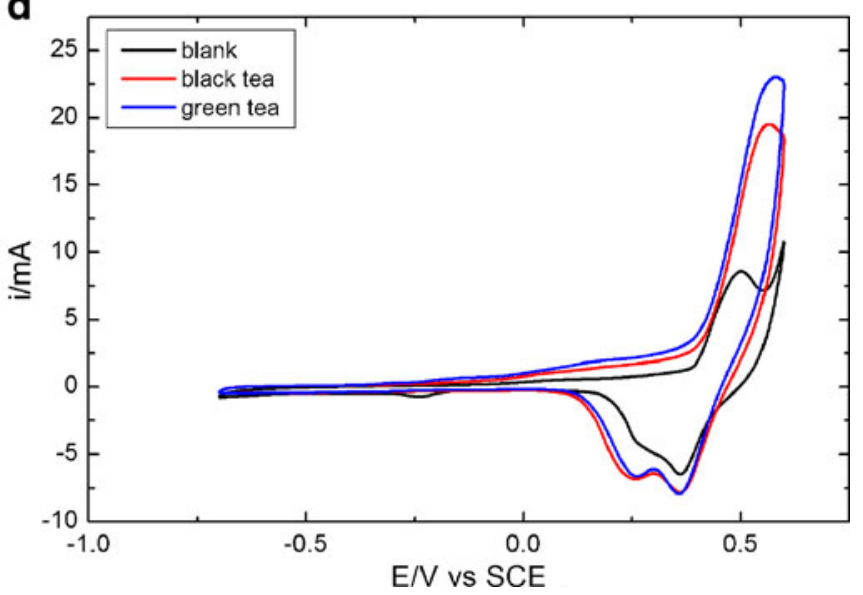

$\mathrm{Bi}_{2} \mathrm{O}_{3}$-modified nickel foam electrode performed as the working electrode. $\mathbf{d} \mathrm{ZnO}$-modified nickel foam electrode performed as the working electrode 
relative intensities of the peaks due to the interactions occurring between the electrode and the compounds solved in the solution studied. Obviously, the numerical differences of peak currents in Fig. 2a and $\mathrm{c}$ are larger than those in Fig. $2 b$ and $d$.

\section{Principal Component Analysis on CV Signals}

In this work, we examined the discriminative ability of different electrodes in distinguishing both green and black teas. Considering that tea infusion has a much complex composition, which may result in the overlapped information, a multivariate data analysis was brought to solve this problem. Principal component analysis (PCA) makes it possible to extract maximum variation information from the high-dimension data matrix with several principal components. PCA is one of the most common data reconstruction and dimensional reduction method, therefore, PCA was performed on the electronic data (Quansheng Chenciq* and Saritporn 2008). In the case of $\mathrm{CV}$, since the anodic wave is more reproducible, the decision taken was to use the anodic wave (which presents an enhanced performance) as the input data.

The PCA score plot corresponding to the nickel foam electrode modified with $\mathrm{SnO}_{2}$ (Fig. 3a) or $\mathrm{TiO}_{2}$ (Fig. 3b) is shown. By analyzing the data exhibited in Fig. 3a, it is possible to see a slight overlap between the green and black teas, with the first two principal components using $79.2 \%$ of the total information. A similar result can be obtained from Fig. $3 b$, with $87.1 \%$ of its total information. In addition, it has to be noticed that Lapsang, one kind of black tea, appears somehow in the overlapping parts of both Fig. $3 a$ and $b$.

The PCA score plots derived from the $\mathrm{Bi}_{2} \mathrm{O}_{3}$ (Fig. 3c) or $\mathrm{ZnO}$ (Fig. 3d) modified nickel foam electrode show a similar distribution of clusters to that displayed in Fig. 3a and b, even if the results were visibly improved, with almost total separation of clusters, including the Lapsang.
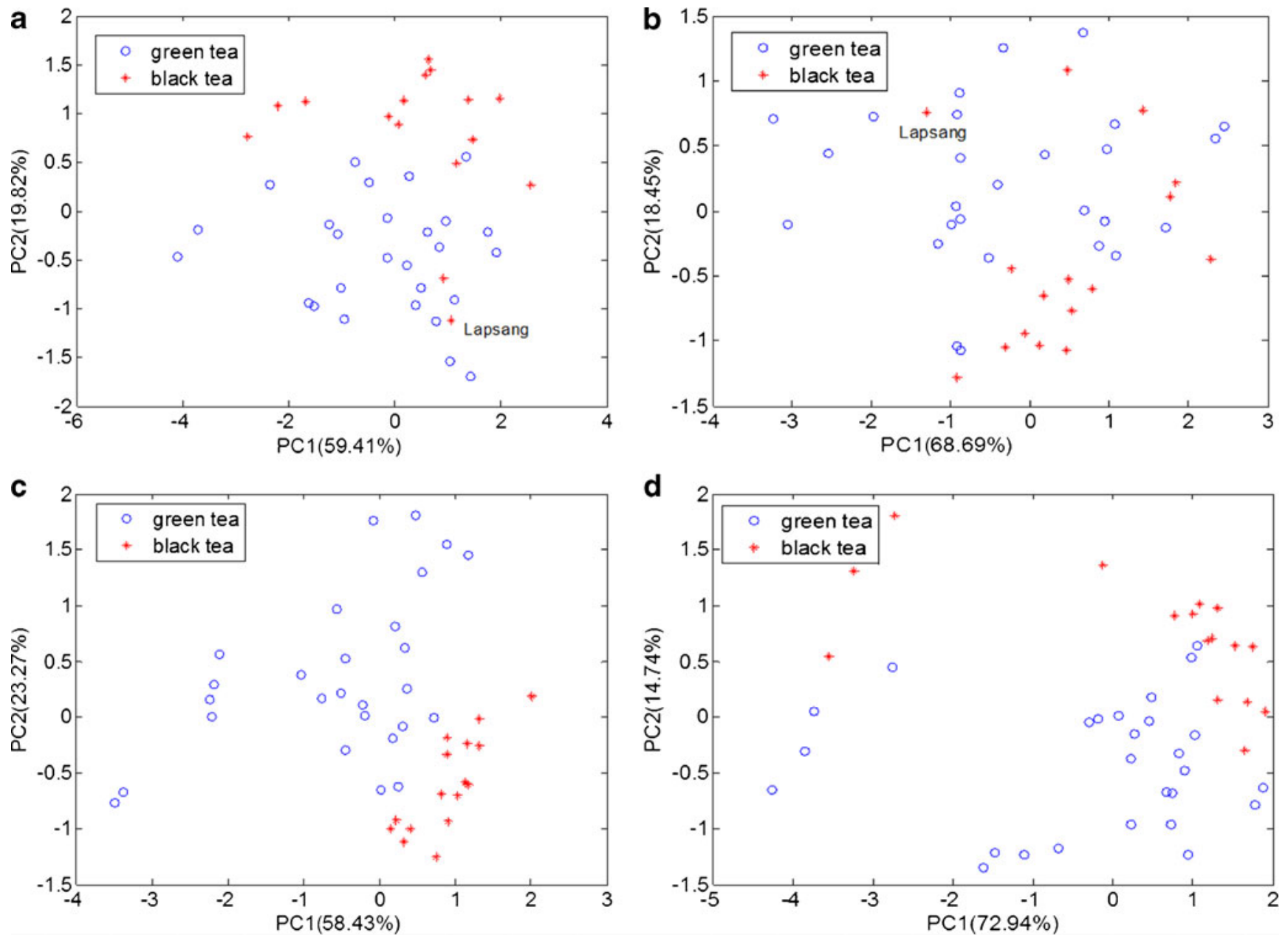

Fig. 3 PCA score plots (two dimensions) of teas. a $\mathrm{SnO}_{2}$-modified nickel foam electrode performed as the working electrode. b $\mathrm{TiO}_{2}$ modified nickel foam electrode performed as the working electrode.

(c) $\mathrm{Bi}_{2} \mathrm{O}_{3}$-modified nickel foam electrode performed as the working electrode. d $\mathrm{ZnO}$-modified nickel foam electrode performed as the working electrode 
Moreover, throughout these four figures, there has been an obvious tendency that the distances between black tea samples are much closer than those of green tea samples in the PCA plots. The reason accounting for this phenomenon was that the main producing areas of black tea were concentrated in three provinces while green tea samples were distributed in eight provinces in this study. Therefore, it was clear that the geographical condition had an influence on the characteristics of the teas, and similar results were reported in previous works (He et al. 2009; Ye et al. 2012).

In the above-presented results, a non-supervised multivariate technique as PCA has demonstrated that this class of electrodes possesses a valuable power for the discrimination of different teas and even portrays the samples' regional distinctions. In particular, such electrodes have a certain degree of similarity, for example, Fig. 3a and b both identify Lapsang improperly, in contrast, Fig. 3c's and d's better classifications reveal the differences among these electrodes. However, considering that the PCA only provides a visualization of the grouping of samples, a mathematic classifier was needed to perform the pattern recognition. In particular, a support vector machine was built to attain a qualitative classification of the teas.

\section{Support Vector Machine Analysis}

Besides the principal component analysis, a supervised method, the support vector machine, was applied to develop the classifier model for the analyzed teas. In SVM, the holdout method of cross validation was employed to randomly divide the dataset into two parts: a training set of $60 \%$ and a testing set of $40 \%$. To achieve a good discrimination performance, determination of appropriate input feature subset and optimization of SVM parameters were the crucial problems to be solved. Firstly, the input data matrices were dimension-reduced by the principal component analysis, PCs that occupied $98 \%$ of the total information were applied as the input datasets for the SVM classifier. Secondly, a grid search technique with fivefold cross-validation was employed to obtain the optimal parameter values of the classifier. Separately, the linear kernel has no special parameters while the polynomial kernel and the Gaussian radial basis function kernel should both consider $\gamma$. As a whole, two important parameters $c$ and $\gamma$ should be taken into account. The ranges of $c$ within $\left(2^{-8}-2^{8}\right)$ and $\gamma$ within $\left(2^{-8}-2^{8}\right)$ were set. Basically, all the $c$ or pairs of $(c, \gamma)$ were conducted and the one with the best cross-validation accuracy was selected.

After the settlement of these problems, the whole training data was evaluated to generate the final classifier. To get a stable and reliable result, the whole dataset was randomly divided 50 times and calculated 50 times. Finally, the three kernel functions were all employed independently and compared to determine which kernel was the most suitable for this system.

The overall averaged result is exhibited in Fig. 4, which is a clustered bar graph wherein the identification rate is plotted with different kernel functions (no. 1 represents the linear kernel, while no. 2 and no. 3 stand for the polynomial kernel and the RBF kernel, respectively) and clustered by four metallic oxide-modified electrodes. In Fig. 4a and b, it is obvious that each of the bars attains a higher identification rate than $80 \%$, what's more, the third bar which is on behalf of the bismuth oxide-modified electrode achieves the highest in all the clusters. The above results indicate that this class of cyclic voltammograms combined with advanced pattern recognition methods acquires a satisfying prediction precision and a more digital identification of different teas.

In the mean time, the type of kernel has some impact on the structure of the obtained SVM, thus, in this work three commonly used kernels were conducted and compared, details are shown in Table 1. Besides the classification accuracies of the dataset, specificity and sensitivity values have also been calculated to provide better statistical description of the model
Fig. 4 Effects of different kernel functions on identification rate for each SVM classifier. a training dataset, $\mathbf{b}$ testing dataset
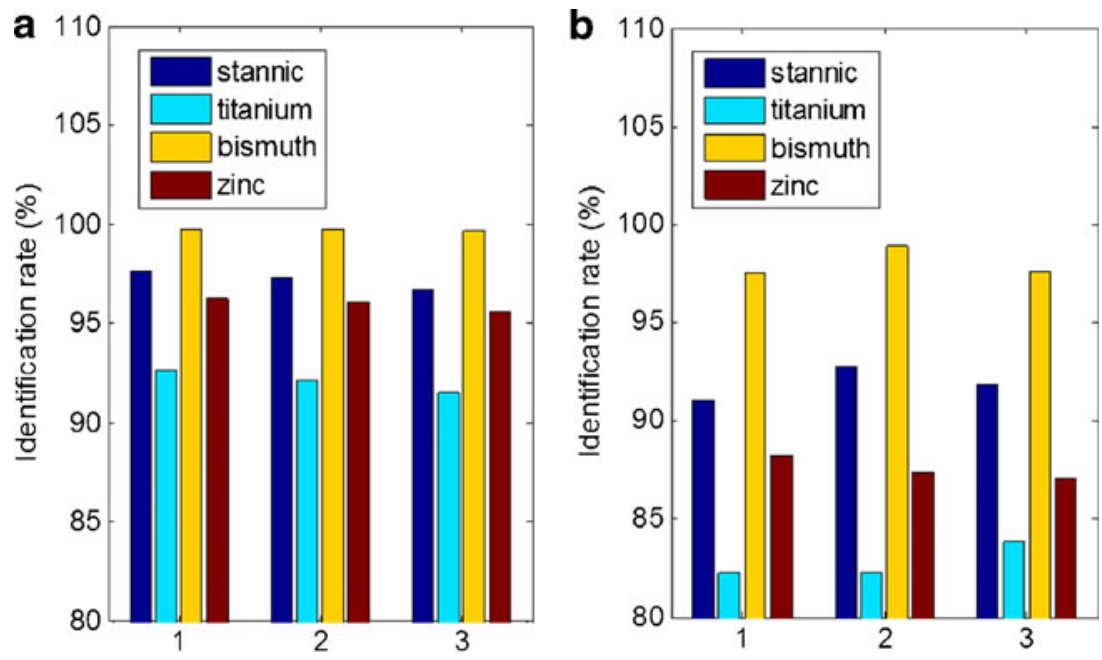
Table 1 Results of the SVM classification with the polynomial kernel using $\mathrm{Bi}_{2} \mathrm{O}_{3}$-modified electrode

\begin{tabular}{|c|c|c|c|c|c|c|}
\hline $\begin{array}{l}\text { Kernel } \\
\text { function }\end{array}$ & $\begin{array}{l}\text { Number of } \\
\text { training data }\end{array}$ & $\begin{array}{l}\text { Classification accuracy of } \\
\text { training data }(\%)\end{array}$ & $\begin{array}{l}\text { Number of } \\
\text { test data }\end{array}$ & $\begin{array}{l}\text { Classification accuracy of } \\
\text { test data }(\%)\end{array}$ & $\begin{array}{l}\text { Sensitivity } \\
(\%)\end{array}$ & $\begin{array}{l}\text { Specificity } \\
(\%)\end{array}$ \\
\hline Linear & 27 & $99.70 \pm 1.02^{\mathrm{a}}$ & 16 & $97.50 \pm 3.18$ & 99.33 & 94.45 \\
\hline Polynomial & 27 & $99.70 \pm 1.02$ & 16 & $98.75 \pm 2.86$ & 99.20 & 94.67 \\
\hline $\mathrm{RBF}$ & 27 & $99.70 \pm 1.02$ & 16 & $97.50 \pm 3.18$ & 98.72 & 95.47 \\
\hline
\end{tabular}

${ }^{\mathrm{a}}$ Mean $\pm \mathrm{RSD}$

performance (Boulesteix et al. 2008). As we have said in the above statements, $\mathrm{Bi}_{2} \mathrm{O}_{3}$-modified electrode gets the best performance, and from Table 1, it can be observed that the prediction performance of the SVM classifier using the polynomial kernel is slightly better than using the other two kernels, with a training accuracy of $99.70 \pm 1.02 \%$ and a testing accuracy of $98.75 \pm 2.86 \%$.

\section{Data Fusion of Multi-sensors}

Furthermore, to make sure whether the accumulated responses from the sensors could improve the final results, several different combinations of sensors have been conducted. As we have mentioned above, $\mathrm{Bi}_{2} \mathrm{O}_{3}$-modified electrode got the best performance among the four single electrodes, so it was fixed in all the array groups. The SVM classifier with polynomial kernel was employed for all the newly combined information, and the result is shown in Table 2.

In the two electrode systems, $\mathrm{Bi}_{2} \mathrm{O}_{3}$ plus $\mathrm{TiO}_{2}, \mathrm{SnO}_{2}$, or $\mathrm{ZnO}$ array shows better classification accuracy than the original $\mathrm{TiO}_{2}, \mathrm{SnO}_{2}$, or $\mathrm{ZnO}$ system; however, the newly combined arrays do not get as high classification accuracies as a single $\mathrm{Bi}_{2} \mathrm{O}_{3}$-modified electrode gets. For the three or four electrode system, a similar result has emerged.

In total, the single $\mathrm{Bi}_{2} \mathrm{O}_{3}$-modified electrode got the best performance in all the groups and the combination of sensors did not show evident improvement in the results. Usually, a vast amount of data is of high complexity ( $\mathrm{Li}$ et al. 2012), for example, there will be outliers or a large number of irrelevant variables. The existence of outliers caused by experimental errors or other uncontrolled factors would make a predictive model misleading, and a large number of irrelevant variables would even interfere with the problem under investigation. In this work, the four electrodes have gotten such a large similarity that their complementary information is limited, thus, the more redundant data may result in worse results.

\section{The Analysis of $\mathrm{Bi}_{2} \mathrm{O}_{3}$-Modified Nickel Foam Electrode}

The most common polyphenols in tea are catechins, which could reach concentration levels of $30 \%$ of the dry material. In the electrochemical reaction process, the catechin is

Table 2 Results of the SVM classification with the polynomial kernel using different groups of electrodes

\begin{tabular}{|c|c|c|c|c|c|c|}
\hline Data set & $\begin{array}{l}\text { Number of } \\
\text { training data }\end{array}$ & $\begin{array}{l}\text { Classification accuracy of } \\
\text { training data }(\%)\end{array}$ & $\begin{array}{l}\text { Number of } \\
\text { test data }\end{array}$ & $\begin{array}{l}\text { Classification accuracy } \\
\text { of test data (\%) }\end{array}$ & $\begin{array}{l}\text { Sensitivity } \\
(\%)\end{array}$ & $\begin{array}{l}\text { Specificity } \\
(\%)\end{array}$ \\
\hline $\mathrm{Bi}_{2} \mathrm{O}_{3}$ plus $\mathrm{TiO}_{2}$ & 27 & $98.22 \pm 2.05^{\mathrm{a}}$ & 16 & $97.88 \pm 3.32$ & 99.67 & 94.89 \\
\hline $\mathrm{Bi}_{2} \mathrm{O}_{3}$ plus $\mathrm{SnO}_{2}$ & 27 & $97.19 \pm 1.96$ & 16 & $97.63 \pm 3.64$ & 97.67 & 97.40 \\
\hline $\mathrm{Bi}_{2} \mathrm{O}_{3}$ plus $\mathrm{ZnO}$ & 27 & $97.56 \pm 1.58$ & 16 & $95.63 \pm 4.81$ & 96.80 & 93.00 \\
\hline $\begin{array}{l}\mathrm{Bi}_{2} \mathrm{O}_{3} \text { plus } \mathrm{TiO}_{2} \text { and } \\
\mathrm{SnO}_{2}\end{array}$ & 27 & $97.63 \pm 2.03$ & 16 & $96.25 \pm 3.94$ & 96.33 & 96.20 \\
\hline $\begin{array}{l}\mathrm{Bi}_{2} \mathrm{O}_{3} \text { plus } \mathrm{TiO}_{2} \text { and } \\
\quad \mathrm{ZnO}\end{array}$ & 27 & $97.78 \pm 1.93$ & 16 & $95.00 \pm 4.61$ & 96.40 & 92.00 \\
\hline $\begin{array}{l}\mathrm{Bi}_{2} \mathrm{O}_{3} \text { plus } \mathrm{SnO}_{2} \text { and } \\
\quad \mathrm{ZnO}\end{array}$ & 27 & $97.78 \pm 1.88$ & 16 & $94.13 \pm 5.27$ & 96.20 & 89.67 \\
\hline $\begin{array}{l}\mathrm{Bi}_{2} \mathrm{O}_{3} \text { plus } \mathrm{TiO}_{2} \text { and } \\
\mathrm{SnO}_{2} \text { and } \mathrm{ZnO}\end{array}$ & 27 & $98.00 \pm 1.98$ & 16 & $94.50 \pm 5.12$ & 95.60 & 93.67 \\
\hline $\mathrm{TiO}_{2}$ & 27 & $91.41 \pm 5.27$ & 16 & $84.00 \pm 11.27$ & 90.40 & 80.33 \\
\hline $\mathrm{SnO}_{2}$ & 27 & $96.89 \pm 2.93$ & 16 & $91.88 \pm 6.49$ & 99.00 & 90.67 \\
\hline $\mathrm{Bi}_{2} \mathrm{O}_{3}$ & 27 & $99.70 \pm 1.02$ & 16 & $98.75 \pm 2.86$ & 99.20 & 94.67 \\
\hline $\mathrm{ZnO}$ & 27 & $95.78 \pm 3.99$ & 16 & $87.13 \pm 6.71$ & 90.80 & 82.33 \\
\hline
\end{tabular}

${ }^{\mathrm{a}}$ Mean \pm RSD 
oxidized to o-quinone, the mechanism of electrochemical oxidation could be expressed as follows:<smiles>[R]c1ccc([O-])c([O-])c1</smiles>

Recently, metallic oxide nanoparticle-modified electrodes have obtained much attention due to their unusual physical and chemical characteristics. Bismuth oxide, which is known as an important transition metal oxide, has been used for a range of applications (Hwang et al. 2008; Li and Yan 2009; Zidan et al. 2011,2010) because of its special energy band gap and photoconductivity. In this system, the electrode reaction of $\mathrm{Bi}_{2} \mathrm{O}_{3}$ is:

$\mathrm{Bi}_{2} \mathrm{O}_{3}+3 \mathrm{H}_{2} \mathrm{O}+6 \mathrm{e}^{-} \Leftrightarrow 2 \mathrm{Bi}+6 \mathrm{OH}^{-}$

In addition, previous literature has also demonstrated that bismuth oxide-modified electrode has a special sensitivity in detecting paracetamol (Zidan et al. 2011), which is a small molecule with a skeleton of phenol. While in this work, as it has been said previously, the main difference of green and black teas is their different concentrations in phenolic compounds. Therefore, the best performance that $\mathrm{Bi}_{2} \mathrm{O}_{3}$-modified electrode gets among other metallic oxides may probably result from bismuth oxidemodified electrode's good sensitivity in identifying phenol substances.

\section{Conclusion}

Cyclic voltammograms in combination with chemometric methods of PCA and SVM were applied for the discrimination of different teas. In particular, four different metallic oxide-modified electrodes were studied, among them $\mathrm{Bi}_{2} \mathrm{O}_{3}$ modified electrode showed the best classification capabilities. Experimental results also revealed that SVM using the polynomial kernel performed the best in obtaining a specific determination.

In conclusion, the approach presented in this work, based on the SVM network, has high potential to evaluate tea sorts. Since our sensor is easy to implement, a simple and rapid classification may be obtained at a low cost, and the method may find applications in the food industry for the identification of other products.

Acknowledgments This work was financially supported by the National Nature Foundation Committee of People's Republic of China (grant nos. 21275164, 21075138). The studies meet the approval of the university's review board. The study is approved by the review board of Central South University.
Conflict of interest Nian Liu declares that she has no conflict of interest. Yizeng Liang declares that he has no conflict of interest. Jun Bin declares that he has no conflict of interest. Zhimin Zhang declares that he has no conflict of interest. Jianhua Huang declares that he has no conflict of interest. This article does not contain any studies with human or animal subjects.

Open Access This article is distributed under the terms of the Creative Commons Attribution License which permits any use, distribution, and reproduction in any medium, provided the original author(s) and the source are credited.

\section{References}

Bhondekar AP, Kaur R, Kumar R, Vig R, Kapur P (2011) A novel approach using dynamic social impact theory for optimization of impedance-tongue (iTongue). Chemometr Intell Lab Syst 109(1):65-76

Boulesteix A-L, Strobl C, Augustin T, Daumer M (2008) Evaluating microarray-based classifiers: an overview. Cancer Informat 6:77

Brereton RG, Lloyd GR (2010) Support vector machines for classification and regression. Analyst 135(2):230-267

Burges CJC (1998) A tutorial on support vector machines for pattern recognition. Data Min Knowl Disc 2(2):121-167

Chang CC, Lin CJ (2011) LIBSVM: a library for support vector machines. ACM Trans Intell Syst Technol| (TIST) 2(3):27

Ciosek P, Brudzewski K, Wróblewski W (2006) Milk classification by means of an electronic tongue and Support Vector Machine neural network. Meas Sci Technol 17(6):1379

del Valle M (2012) Sensor arrays and electronic tongue systems. I J Electrochem

Dufresne CJ, Farnworth ER (2001) A review of latest research findings on the health promotion properties of tea. J Nutr Biochem 12(7):404-421

Escuder-Gilabert L, Peris M (2010) Review: highlights in recent applications of electronic tongues in food analysis. Anal Chim Acta 665(1):15-25

Ferreira M, Varela H, Torresi RM, Tremiliosi-Filho G (2006) Electrode passivation caused by polymerization of different phenolic compounds. Electrochim Acta 52(2):434-442

Freire RS, Ferreira M, Durán N, Kubota LT (2003) Dual amperometric biosensor device for analysis of binary mixtures of phenols by multivariate calibration using partial least squares. Anal Chim Acta 485(2):263-269

Gay Martín M, de Saja JA, Muñoz R, Rodríguez-Méndez ML (2012) Multisensor system based on bisphthalocyanine nanowires for the detection of antioxidants. Electrochim Acta 68:88-94

Ghasemi-Varnamkhasti M, Rodríguez-Méndez ML, Mohtasebi SS et al (2012) Monitoring the aging of beers using a bioelectronic tongue. Food Control 25(1):216-224

Gutés A, Céspedes F, Alegret S, Del Valle M (2005) Determination of phenolic compounds by a polyphenol oxidase amperometric biosensor and artificial neural network analysis. Biosens Bioelectron 20(8):1668-1673

Gutes A, Ibanez A, Cespedes F, Alegret S, Del Valle M (2005) Simultaneous determination of phenolic compounds by means of an automated voltammetric "electronic tongue". Anal Bioanal Chem 382(2):471-476

Gutiérrez JM, Gutés A, Céspedes F, del Valle M, Muñoz R (2008) Wavelet neural networks to resolve the overlapping signal in the voltammetric determination of phenolic compounds. Talanta 76(2):373-381

Harbowy ME, Balentine DA, Davies DAP, Cai DY (1997) Tea chemistry. Crit Rev Plant Sci 16(5):415-480 
He W, Hu X, Zhao L et al (2009) Evaluation of Chinese tea by the electronic tongue: correlation with sensory properties and classification according to geographical origin and grade level. Food Res Int 42(10):1462-1467

Hu Hai XW, Yuan J, Shi J, Chen M, Shangguan W* (2007) Preparations of $\mathrm{TiO}_{2}$ film coated on foam nickel substrate by sol-gel processes and its photocatalytic activity for degradation of acetaldehyde. J Environ Sci 19:80-85

Hwang GH, Han WK, Park JS, Kang SG (2008) An electrochemical sensor based on the reduction of screen-printed bismuth oxide for the determination of trace lead and cadmium. Sensors Actuators B Chem 135(1):309-316

Ivarsson P, Holmin S, Höjer NE, Krantz-Rülcker C, Winquist F (2001) Discrimination of tea by means of a voltammetric electronic tongue and different applied waveforms. Sensors Actuators B Chem 76(1):449-454

Jaimez J, Fente C, Vazquez B et al (2000) Application of the assay of aflatoxins by liquid chromatography with fluorescence detection in food analysis. J Chromatogr A 882(1):1-10

Kikkawa Y, Toko K, Yamafuji K (1993) Taste sensing of tomatoes with a multichannel taste sensor (S \& M 0129). Sensor Mater 5:83-83

Kilmartin PA, Hsu CF (2003) Characterisation of polyphenols in green, oolong, and black teas, and in coffee, using cyclic voltammetry. Food Chem 82(4):501-512

Kumar R, Bhondekar AP, Kaur R, Vig S, Sharma A, Kapur P (2012) A simple electronic tongue. Sensors Actuators B Chem

Laura Escuder-Gilaberta MP (2010) Review: highlights in recent applications of electronic tongues in food analysis. Anal Chim Acta 665:15-25

Li L, Yan B (2009) $\mathrm{BiVO}<$ sub $>4</$ sub $>/ \mathrm{Bi}<$ sub $>2</$ sub $>$ O $<$ sub $>$ $3</$ sub $>$ submicrometer sphere composite: microstructure and photocatalytic activity under visible-light irradiation. J Alloy Compd 476(1):624-628

Li H, Liang Y, Xu Q (2009) Support vector machines and its applications in chemistry. Chemometr Intell Lab Syst 95(2): 188-198

Li H-D, Liang Y-Z, Cao D-S, Xu Q-S (2012) Model-population analysis and its applications in chemical and biological modeling. TrAC Trends Anal Chem 38:154-162

Lvova L, Kim SS, Legin A et al (2002) All-solid-state electronic tongue and its application for beverage analysis. Anal Chim Acta 468(2):303-314

Lvova L, Legin A, Vlasov Y, Cha GS, Nam H (2003) Multicomponent analysis of Korean green tea by means of disposable all-solid-state potentiometric electronic tongue microsystem. Sensors Actuators B Chem 95(1):391-399

McKay DL, Blumberg JB (2002) The role of tea in human health: an update. J Am Coll Nutr 21(1):1-13

Nielsen SS (2010) Food analysis. Springer, New York

Nollet LML (2000) Food analysis by HPLC, vol 100. CRC, Boca Raton

Novak I, Šeruga M, Komorsky-Lovrić Š (2009) Electrochemical characterization of epigallocatechin gallate using square-wave voltammetry. Electroanal 21(9):1019-1025

Novakowski W, Bertotti M, Paixão TRLC (2011) Use of copper and gold electrodes as sensitive elements for fabrication of an electronic tongue: discrimination of wines and whiskies. Microchem J 99(1):145-151

Otto M, Thomas J (1985) Model studies on multiple channel analysis of free magnesium, calcium, sodium, and potassium at physiological concentration levels with ion-selective electrodes. Anal Chem 57(13):2647-2651
Quansheng Chen* JZ, Saritporn V (2008) Identification of the green tea grade level using electronic tongue and pattern recognition. Food Res Int 41:500-504

Riul A Jr, Dantas CA, Miyazaki CM, Oliveira ON Jr (2010) Recent advances in electronic tongues. Analyst 135(10):2481-2495

Roginsky V, Barsukova T, Hsu CF, Kilmartin PA (2003) Chainbreaking antioxidant activity and cyclic voltammetry characterization of polyphenols in a range of green, oolong, and black teas. J Agr Food Chem 51(19):5798-5802

Scampicchio M, Benedetti S, Brunetti B, Mannino S (2006) Amperometric electronic tongue for the evaluation of the tea astringency. Electroanal 18(17):1643-1648

Shawe-Taylor J, Cristianini N (2000) An introduction to support Vector Machines: and other kernel-based learning methods. Cambridge University Press 29:136

Simo C, Barbas C, Cifuentes A (2005) Capillary electrophoresis-mass spectrometry in food analysis. Electrophoresis 26(7-8):1306-1318

Smola AJ, Schölkopf B (1998) Learning with kernels: citeseer

Tian SY, Deng SP, Chen ZX (2007) Multifrequency large amplitude pulse voltammetry: a novel electrochemical method for electronic tongue. Sensors Actuators B Chem 123(2):1049-1056

Toko K (2000) Taste sensor. Sensors Actuators B Chem 64(1):205-215

Vapnik V (1998) Statistical learning theory. Wiley, New York

Vlasov YG, Bychkov E, Legin A (1994) Chalcogenide glass chemical sensors: research and analytical applications. Talanta 41(6):10591063

Vlasov YG, Legin A, Rudnitskaya A, d'Amico A, Natale CD (1997) Chemical analysis of multicomponent aqueous solutions using a system of nonselective sensors and artificial neural networks. J Anal Chem 52(11):1087-1092

Vlasov YG, Legin A, Rudnitskaya A, D'Amico A, Di Natale C (2000) «Electronic tongue»-new analytical tool for liquid analysis on the basis of non-specific sensors and methods of pattern recognition. Sensors Actuators B Chem 65(1):235-236

Wang G, He X, Li Z, Fang B, Zhang X, Wang L (2012) Application of gold nanoparticles $/ \mathrm{TiO}<\mathrm{sub}>2</$ sub $>$ modified electrode for the electrooxidative determination of catechol in tea samples. Food Chem 135:446-451

Webb AR (2003) Statistical pattern recognition. Wiley

Wei Z, Wang J (2011) Detection of antibiotic residues in bovine milk by a voltammetric electronic tongue system. Anal Chim Acta 694(1):46-56

Winquist F, Wide P, Lundström I (1997) An electronic tongue based on voltammetry. Anal Chim Acta 357(1-2):21-31

Winquist F, Holmin S, Krantz-Rülcker C, Wide P, Lundström I (2000) A hybrid electronic tongue. Anal Chim Acta 406(2):147-157

Winquist F, Bjorklund R, Krantz-Rülcker C, Lundström I, Östergren K, Skoglund T (2005) An electronic tongue in the dairy industry. Sensors Actuators B Chem 111:299-304

Wold S, Esbensen K, Geladi P (1987) Principal component analysis. Chemometr Intell Lab Syst 2:37-52

Ye N, Zhang L, Gu X (2012) Discrimination of green teas from different geographical origins by using HS-SPME/GC-MS and pattern recognition methods. Food Anal Methods 1-5

Zidan M, WeeTee T, Zainal Z, Abdullah AH, Goh JK (2010) Electrocatalytic oxidation of ascorbic acid mediated by lithium doped microparticles Bi2O3/MWCNT modified glassy carbon electrode. Int J Electrochem Sci 5:501-508

Zidan M, Tee TW, Abdullah AH, Zainal Z, Kheng GJ (2011) Electrochemical oxidation of paracetamol mediated by nanoparticles bismuth oxide modified glassy carbon electrode. Int J Electrochem Sci 6:279-288 